Pacific Journal of Mathematics

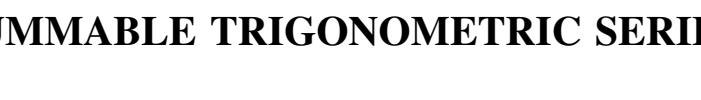




\title{
SUMMABLE TRIGONOMETRIC SERIES
}

\author{
R. D. JAMES
}

1. Introduction. One of the problems in the theory of trigonometric series in the form

$$
\frac{1}{2} a_{0}+\sum_{n=1}^{\infty}\left(a_{n} \cos n x+b_{n} \sin n x\right)=\sum_{n=0}^{\infty} a_{n}(x)
$$

is that of suitably defining a process of integration such that, if the series (1.1) converges to a function $f(x)$, then $f(x)$ is integrable and the coefficients $a_{n}, b_{n}$ are given in Fourier form. The problem has been solved by Denjoy [3], Verblunsky [10], Marcinkiewicz and Zygmund [8], Burkill [1], [2], and James [6]. In Verblunsky's paper and in Burkill's first paper, additional hypotheses other than the convergence of (1.1) were made, and in all the papers some modification of the form of the Fourier formulas was necessary:

An extension of the problem is to consider series that are summable $(C, k), k \geqq 1$. This has been solved by Wolf [11] when the sum function is Perron integrable. The problem of defining a process of integration which may be applied to any series summable $(C, k)$ may be solved if an additional condition involving the conjugate series

$$
\sum_{n=1}^{\infty}\left(a_{n} \sin n x-b_{n} \cos n x\right)=-\sum_{n=1}^{\infty} b_{n}(x)
$$

is imposed. With this extra condition, it is proved, in $\S 2$, that the formal product of $\cos p x$ or $\sin p x$ and a series summable $(C, k)$ to $f(x)$ is also summable $(C, k)$ to $f(x) \cos p x$ or $f(x) \sin p x$.

In $\S 3$, some properties of integrated series are discussed and then, in $\S 4$, it is shown that the generalized $P^{k+2}$-integral [7] integrates any trigonometric series summable $(C, k)$ and satisfying the extra condition. In addition, the coefficients are given by a natural modification of the Fourier formulas. These are the principal results of the paper. They were described briefly for the special case $k=2$ in the author's invited address at the 1954 Summer Meeting of the American Mathematical Society.

It is also possible to improve the results slightly and only require summability for all $x$ in $[0,2 \pi]$ with the possible exception of a countable set. This requires a minor modification in the definition of the $P^{k+2}$-integral and these changes are indicated in $\S \S 5$ and 6 .

Received December 20, 1954. Presented to the American Mathematical Society, June 19, 1954. 
2. Formal multiplication of summable trigonometric series. Following the notation of Hardy $[4, \S 5.4]$, let

$$
\begin{array}{ll}
A_{n}^{0}(x)=A_{n}(x)=\sum_{r=0}^{n} a_{r}(x), & A_{n}^{k}(x)=\sum_{r=0}^{n} A_{r}^{k-1}(x), \\
B_{n}^{0}(x)=B_{n}(x)=\sum_{r=1}^{n} b_{r}(x), & B_{n}^{k}(x)=\sum_{r=1}^{n} B_{r}^{k-1}(x),
\end{array}
$$

where

$$
\begin{aligned}
a_{\vartheta}(x)= & a_{0} / 2, a_{n}(x)=a_{n} \cos n x+b_{n} \sin n x, & \\
& b_{n}(x)=b_{n} \cos n x-a_{n} \sin n x, & n \geqq 1,
\end{aligned}
$$

and let $E_{n}^{k}=(n+k) ! / n ! k !$. If $A_{n}^{k}(x) / E_{n}^{k} \rightarrow f(x)$ as $n \rightarrow \infty$, the series (1.1) is said to be summable $(C, k)$ to $f(x)$ and the notation is

$$
\sum a_{n}(x)=f(x) \quad(C, k) .
$$

The formal product of $g(x)=\lambda \cos p x+\mu \sin p x, p \geqq 1$, and the series (1.1) is the series obtained by multiplying each term by $g(x)$, replacing the trigonometric products by sums of cosines and sines, and rearranging the terms in the form

$$
\sum_{n=0}^{\infty}\left(u_{n} \cos n x+v_{n} \sin n x\right)=\sum_{n=0}^{\infty} u_{n}(x)
$$

where

$$
u_{0}(x)=\frac{1}{2} u_{0}=\frac{1}{2}\left(\lambda a_{p}+\mu b_{p}\right)
$$

$$
u_{n}(x)=\frac{1}{2}\left[\lambda\left\{a_{n-p}(x)+a_{n+p}(x)\right\}-\mu\left\{b_{n-p}(x)-b_{n+p}(x)\right\}\right],
$$

with the usual convention that $a_{-r}(x)=a_{r}(x), b_{0}(x)=0, b_{-r}(x)=-b_{r}(x)$. Similarly, the formal product of $g(x)$ and the conjugate series (1.2) is

$$
\sum_{n=1}^{\infty}\left(v_{n} \cos n x-u_{n} \sin n x\right)=-\sum_{n=1}^{\infty} v_{n}(x),
$$

where

$$
v_{n}(x)=\frac{1}{2}\left[\lambda\left\{b_{n-p}(x)+b_{n+p}(x)\right\}+\mu\left\{a_{n-p}(x)-a_{n+p}(x)\right\}\right] .
$$

Before proving the main result of this section, it is convenient to find expressions for $U_{n}^{k}(x)$ and $V_{n}^{k}(x)$. It will be seen later that it is suf- 
ficient to consider the case $x=0$. The method is similar to that of Zygmund [12], who proved the analogous result for Abel (or Poisson) summability.

The definitions (2.1), with $x=0$, are equivalent to the identity

$$
\sum_{n=0}^{\infty}\left(U_{n}^{k}-\lambda A_{n}^{k}\right) z^{n+p}=(1-z)^{-k-1} z^{p}\left\{\frac{1}{2}\left(u_{0}-\lambda a_{0}\right)+\sum_{n=1}^{\infty}\left(u_{n}-\lambda a_{n}\right) z^{n}\right\} .
$$

When the formulas (2.3), (2.4), with $x=0$, are substituted in the right side of (2.5), the coefficient of $\lambda / 2$ may be written in the form

$$
\begin{aligned}
(1-z)^{-k-1} & {\left[\left(1-z^{p}\right)^{2}\left(\frac{1}{2} a_{0}+\sum_{n=1}^{\infty} a_{n} z^{n}\right)\right.} \\
& \left.-\left\{\frac{1}{2} a_{0}\left(1-z^{2 p}\right)+\sum_{n=1}^{\infty} a_{n}\left(z^{n}-z^{2 p-n}\right)\right\}\right],
\end{aligned}
$$

and the coefficient of $\mu / 2$,

$$
(1-z)^{-k-1}\left[\left(1-z^{2 p}\right) \sum_{n=1}^{\infty} b_{n} z^{n}-\sum_{n=1}^{\infty} b_{n}\left(z^{n}-z^{2 p-n}\right)\right] .
$$

The first series in (2.6) becomes

$$
\left\{\sum_{n=0}^{2 p-2} c_{n} z^{n}\right\}\left\{(1-z)^{-k+1}\left(\frac{1}{2} a_{0}+\sum_{n=1}^{\infty} a_{n} z^{n}\right)\right\}=\left\{\sum_{n=0}^{2 p-2} c_{n} z^{n}\right\}\left\{\sum_{n=0}^{\infty} A_{n}^{k-2} z^{n}\right\},
$$

where

$$
c_{n}=\left\{\begin{array}{lr}
n+1 & 0 \leqq n \leqq p-1, \\
2 p-n-1 & p \leqq n \leqq 2 p-2 .
\end{array}\right.
$$

The second series in (2.6) is

$$
P(z)(1-z)^{-k}=P(z) \sum_{n=0}^{\infty} E_{n}^{k-1} z^{n},
$$

where $P(z)$ is a polynomial of degree $2 p-1$ at most. Similarly, the series in (2.7) become

$$
\left\{\sum_{n=0}^{2 p-1} z^{n}\right\}\left\{\sum_{n=1}^{\infty} B_{n}^{k-1} z^{n}\right\} \text { and } Q(z) \sum_{n=1}^{\infty} E_{n}^{k-1} z^{n},
$$

where $Q(z)$ is a polynomial of degree $2 p-2$ at most.

Since $E_{n}^{k-1} \sim n^{k-1} /(k-1)$ !, it follows by equating coefficients in (2.5) and (2.8), (2.9), (2.10), that

$$
U_{n}^{k}-\lambda A_{n}^{k}=\frac{1}{2} \lambda \sum_{r=n-p+2}^{n+p} c_{n+p-r} A_{r}^{k-2}+\frac{1}{2} \mu \sum_{r=n-p+1}^{n+p} B_{r}^{k-1}+O\left(n^{k-1}\right) .
$$


A similar computation shows that

$$
V_{u}^{k}-\lambda B_{n}^{k}=K_{p} E_{n}^{k}+\frac{1}{2} \lambda \sum_{r=n-p+2}^{n+p} c_{n+p-r} B_{r}^{k-2}-\frac{1}{2} \mu \sum_{r=n-p+1}^{n+p} A_{r}^{k-1}+O\left(n^{k-1}\right),
$$

where

$$
K_{p}=\frac{1}{2} \mu\left(a_{0}+a_{p}+2 \sum_{s=1}^{p-1} a_{s}\right)-\frac{1}{2} \lambda\left(b_{p}+2 \sum_{s=1}^{p-1} b_{s}\right) .
$$

The main result of the section now follows from (2.11) and (2.12). THEOREM 2.1. If

$$
A_{n}^{k-2}\left(x_{\jmath}\right)=o\left(n^{k}\right), \quad B_{n}^{k-1}\left(x_{0}\right)=o\left(n^{k}\right),
$$

then

$$
\begin{gathered}
\sum_{n=0}^{\infty}\left\{u_{n}\left(x_{0}\right)-g\left(x_{0}\right) a_{n}\left(x_{0}\right)\right\}=0 \quad(C, k), \\
U_{n}^{k-2}\left(x_{0}\right)=o\left(n^{k}\right), \quad V_{n}^{k-1}\left(x_{0}\right)=o\left(n^{k}\right) .
\end{gathered}
$$

Proof. Since

$$
a_{n}\left(x_{0}+x\right)=a_{n}\left(x_{0}\right) \cos n x+b_{n}\left(x_{0}\right) \sin n x,
$$

with similar expressions for $b_{n}\left(x_{0}+x\right)$ and $g\left(x_{0}+x\right)$, formula (2.11) is valid with $U_{n}^{k}, \lambda, A_{n}^{k}$, replaced respectively by $U_{n}^{k}\left(x_{0}\right), g\left(x_{0}\right), A_{n}^{k}\left(x_{0}\right)$, and with similar replacements on the right side. Thus,

$$
U_{n}^{k}\left(x_{0}\right)-g\left(x_{0}\right) A_{n}^{k}\left(x_{0}\right)=o\left(n^{k}\right)
$$

and this is equivalent to (2.14).

Similarly, since (2.13) imply

$$
A_{n}^{r-2}\left(x_{0}\right)=o\left(n^{k}\right), \quad B_{n}^{r-1}\left(x_{0}\right)=o\left(n^{k}\right)
$$

for $r<k$, the other conclusions of the theorem follow from (2.11) and (2.12) with $k$ replaced by $k-2$ and $k-1$, respectively.

3. Integrated trigonometric series. In the work of Riemann there are two fundamental results for series (1.1) with coefficients $a_{n}$ and $b_{n}$ tending to zero $[13, \S 11.2]$. These results have been generalized for series in which $a_{n}=o\left(n^{k}\right), b_{n}=o\left(n^{k}\right)$. They involve generalized (symmetric) derivatives $[13, \S 10.41]$ defined successively by

$$
D^{p} F(x)=\lim _{h \rightarrow 0} \theta_{p}(x, h)=\lim _{h \rightarrow 0} \theta_{p}(F ; x, h),
$$

where, for $p=2 m$, 


$$
\frac{h^{2 m}}{(2 m) !} \theta_{2 m}(x, h)=\frac{1}{2}\{F(x+h)+F(x-h)\}-\sum_{s=0}^{m-1} \frac{h^{2 s}}{(2 s) !} D^{2 s} F(x),
$$

and, for $p=2 m+1$,

$$
\frac{h^{2 m+1}}{(2 m+1) !} \theta_{2 m+1}(x, h)=\frac{1}{2}\{F(x+h)-F(x-h)\}-\sum_{s=0}^{m-1} \frac{h^{2 s+1}}{(2 s+1) !} D^{2 s+1} F(x) .
$$

The generalizations of Riemann's results are given in [11, Theorem $B]$ and $[13, \S 10.42]$ and are conveniently stated in terms of the following conditions that may be imposed on the series (1.1):

$$
\begin{gathered}
a_{n}=o\left(n^{k}\right), \quad b_{n}=o\left(n^{k}\right), \\
A_{n}^{k-1}\left(x_{0}\right)=o\left(n^{k}\right), \\
\frac{1}{2} a_{0}+\sum_{n=1}^{\infty} a_{n}\left(x_{0}\right)=f\left(x_{0}\right)
\end{gathered}
$$

THEOREM 3.1. If condition (3.3) is satisfied, then the series obtained by integrating (1.1) formally term-by-term $k+2$ times converges uniformly to a continuous function $F(x)$. If conditions (3.3) and (3.4) are both satisfied, then $D^{k+2-2 r} F\left(x_{0}\right)$ exists for $1 \leqq r \leqq(k+1) / 2$ and

$$
h \theta_{k+2}\left(x_{0}, h\right) \rightarrow 0 \quad \text { as } h \rightarrow 0 .
$$

If conditions (3.3) and (3.5) are both satisfied, then (3.6) holds and, in addition,

$$
\frac{a_{0} x_{0}^{2 r}}{2(2 r) !}+(-1)^{r} \sum_{n=1}^{\infty} \frac{a_{n}\left(x_{0}\right)}{n^{2 r}}=D^{k+2-2 r} F\left(x_{0}\right)
$$

for $0 \leqq r \leqq(k+1) / 2$.

It may happen that the derivatives exist for all $x$ in an interval $(a, b)$. For later purposes it is necessary to know under what conditions no derivative has an ordinary discontinuity in $(a, b)$. A sufficient condition involves the series

$$
\frac{1}{2} a_{0} x-\sum_{n=1}^{\infty} c_{n}(x)
$$

where $c_{n}(x)=b_{n}(x) / n$. This series is, of course, obtained from (1.1) by integrating formally term-by-term.

THEOREM 3.2. If conditions (3.3), (3.4), and

$$
C_{n}^{k-2}\left(x_{0}\right)=o\left(n^{k-1}\right)
$$

are all satisfied for every $x_{0}$ in an interval $(a, b)$, then no derivative 
$D^{k+2-2 r} F(x), \quad 1 \leqq r \leqq(k+1) / 2$, has an ordinary discontinuity in $(a, b)$.

Proof. The function $F(x)$ of Theorem 3.1 is also the function obtained by integrating (3.8) formally term-by-term $k+1$ times. Since conditions (3.3) imply that the coefficients of (3.8) are $o\left(n^{k-1}\right)$, it follows from (3.9) and Theorem 3.1 that each of $h \theta_{k+1}\left(x_{0}, h\right)$ and $h \theta_{k+2}\left(x_{0}, h\right)$ tends to zero as $h \rightarrow 0$. If $k$ is even, definitions (3.1) and (3.2) show that

$$
\begin{aligned}
& \frac{1}{2}\left\{F\left(x_{0}+h\right)-F\left(x_{0}-h\right)\right\}=\sum_{s=0}^{(k-2) / 2} \frac{h^{2 s+1}}{(2 s+1) !} D^{2 s+1} F\left(x_{0}\right)+o\left(h^{k}\right), \\
& \frac{1}{2}\left\{F\left(x_{0}+h\right)+F\left(x_{0}-h\right)\right\}=F\left(x_{0}\right)+\sum_{s=1}^{k / 2} \frac{h^{2 s}}{(2 s) !} D^{2 s} F\left(x_{0}\right)+o\left(h^{k+1}\right),
\end{aligned}
$$

and there are similar results when $k$ is odd.

Hence, by addition and subtraction,

$$
F\left(x_{0} \pm h\right)=F\left(x_{0}\right)+\sum_{r=1}^{k} \frac{( \pm h)^{r}}{r !} D^{r} F\left(x_{0}\right)+o\left(h^{k}\right) .
$$

But this is precisely the definition $[13, \S 10.41]$ of the generalized nonsymmetric derivatives of $F(x)$. Thus $D^{r} F\left(x_{0}\right)=F_{(r)}\left(x_{0}\right)$ and it is known [7, Lemma 8.1] that when $F(x)$ is continuous, then $F_{(r)}(x)$ cannot have an ordinary discontinuity.

4. The expression of an everywhere summable trigonometric series in Fourier form. This section contains the principal results of the paper.

THeOREM 4.1. If the series (1.1) is summable $(C, k)$ for all $x$ to a finite function $f(x)$, and if condition (3.9) holds for all $x$, then $f(x)$ is $P^{k+2}$-integrable [7, Definition 5.1] over $\left(\alpha_{i} ; x\right)$ for every $x$ in $[\alpha, \beta]$. Here $[\alpha, \beta]$ is any finite closed interval and

$$
\alpha \leqq \alpha_{1}<\alpha_{2}<\cdots<\alpha_{k+2} \leqq \beta .
$$

Proof. Since the series is summable $(C, k)$ for all $x$, conditions (3.3) are satisfied $[13, \S 11.11]$ and (3.4) holds for all $x$. It follows from Theorem 3.1 that (3.6) and (3.7) are valid for all $x$. By Theorem 3.2, no derivative $D^{k+2-2 r} F(x), 1 \leqq r \leqq(k+1) / 2$, has an ordinary discontinuity. Let

$$
Q(x)=F(x)-\sum_{i=1}^{k+2} \lambda\left(x ; \alpha_{i}\right) F\left(\alpha_{i}\right),
$$

where

$$
\lambda\left(x ; \alpha_{i}\right)=\prod_{j \neq i}\left(x-\alpha_{j}\right) /\left(\alpha_{i}-\alpha_{j}\right)
$$

is a polynomial of degree $k+1$. Then 


$$
\begin{aligned}
& h \theta_{k+2}(Q ; x, h)=h \theta_{k+2}(F ; x, h), \\
& Q\left(\alpha_{i}\right)=0, \quad i=1, \cdots, k+2, \\
& D^{k+2} Q(x)=D^{k+2} F(x)=f(x) .
\end{aligned}
$$

Thus $Q(x)$ satisfies all the conditions [7, Definition 5.1] for both a major and a minor function of $f(x)$, and $f(x)$ is therefore $P^{k+2}$-integrable over $\left(\alpha_{i} ; x\right)$.

Theorem 4.2. Under the hypotheses of Theorem 4.1, but with (3.9) replaced by

$$
B_{n}^{k-1}(x)=o\left(n^{k}\right) \text { for all } x,
$$

the functions $f(x) \cos p x$ and $f(x) \sin p x, p \geq 1$, are each $P^{k+2}$-integrable over $\left(\alpha_{i} ; x\right)$.

Proof. It is not difficult to see (for example, by the method of proof of [4, Theorem 50]) that (4.2) implies (3.9), so that the hypotheses of Theorem 4.2 are stronger than those of Theorem 4.1.

All the hypotheses of Theorem 2.1 are satisfied for all $x$ so that (2.14) is true. Thus, with $\lambda=1, \mu=0$,

$$
\frac{1}{2} u_{0}+\sum_{n=1} u_{n}(x)=f(x) \cos p x
$$

and $u_{n}=o\left(n^{k}\right), v_{n}=o\left(n^{k}\right)$. By Theorem 3.1, the series obtained by integrating (4.3) formally term-by-term $k+2$ times converges uniformly to a continuous function $G_{p}(x)$ such that (3.6) and (3.7) hold, with $F$ replaced by $G_{p}$, for all $x$.

It remains to show that no derivative of $G_{p}(x)$ has an ordinary discontinuity. The series corresponding to (3.8) is

$$
\frac{1}{2} u_{0} x-\sum w_{n}(x)
$$

where $w_{n}(x)=v_{n}(x) / n$. By (2.15) of Theorem 2.1, $V_{n}^{k-1}(x)=o\left(n^{k}\right)$ and this, as noted above, implies $W_{n}^{k-2}(x)=o\left(n^{k-1}\right)$. Thus, by the proof of Theorem 4.1, $f(x) \cos p x$ is $P^{k+2}$-integrable over $\left(\alpha_{i} ; x\right)$.

By taking $\lambda=0, \mu=1$ in (2.14), it follows in a similar fashion that the same result is true for $f(x) \sin p x$.

COROLLARY. If $k=2 m-2$, let $\gamma_{k}=(2 m) ! /(m !)^{2}$ and let $\left(\alpha_{i}\right)$ be the set

$$
(-2 m \pi, \cdots,-2 \pi, 2 \pi, \cdots, 2 m \pi) \text {. }
$$

If $k=2 m-1$, let $\gamma_{k}=(2 m+1) ! / m !(m+1)$ ! and let $\left(\alpha_{i}\right)$ be the set

$$
(-2 m \pi, \cdots,-2 \pi, 2 \pi, \cdots,(2 m+2) \pi) \text {. }
$$


Then, under the hypotheses of Theorem 4.2, the coefficients of the series (1.1) are given by

$$
\begin{aligned}
& a_{p}=\frac{\gamma_{k}}{2^{k+1} \pi^{k+2}} \int_{\left(\alpha_{i}\right)}^{0} f(x) \cos p x d_{k+2} x, \\
& b_{p}=\frac{\gamma_{k}}{2^{k+1} \pi^{k+2}} \int_{\left(\alpha_{i}\right)}^{0} f(x) \sin p x d_{k+2} x .
\end{aligned}
$$

Proof. Only the case $k=2 m-2$ will be considered, since the details for $k=2 m-1$ are quite similar. It follows from the proof of Theorem 4.1 and the definition of the $P^{2 m}$-integral that, for $\alpha_{m} \leqq x$ $<\alpha_{m+1}$,

$$
(-1)^{m} \int_{\left(\alpha_{i}\right)}^{x} f(x) d_{2 m} x=F(x)-\sum_{i=1}^{2 m} \lambda\left(x ; \alpha_{i}\right) F\left(\alpha_{i}\right)
$$

When $k=2 m-2, F(x)$ may be written in the form $G(x)+H(x)$, where $G(x)=a_{0} x^{2 m} / 2(2 m)$ ! and $H(x)$ is a periodic function with period $2 \pi$. If $\left(\alpha_{i}\right)$ is the set (4.4) and $x=0$, the right side of (4.7) becomes

$$
G(0)-\sum_{i=1}^{2 m} \lambda\left(0 ; \alpha_{i}\right) G\left(\alpha_{i}\right)+H(0)-\sum_{i=1}^{2 m} \lambda\left(0 ; \alpha_{i}\right) H(0) .
$$

The terms involving $G(x)$ are equal to $\Pi_{i=1}^{2 m}\left(-\alpha_{i}\right)$ multiplied by a divided difference [9, Chapter I] of order $2 m$ for the function $G(x)$. Since every divided difference of order $2 m$ for the function $x^{2 m}$ is equal to 1 and the divided difference of the constant $H(0)$ is equal to 0 , the expression (4.8) reduces to

$$
\frac{a_{0}}{2(2 m) !} \prod_{i=1}^{2 m}\left(-\alpha_{i}\right)=\frac{(-1)^{m} a_{0} 2^{k+1} \pi^{k+2}}{\gamma_{k}}
$$

by (4.4). This, together with (4.7) shows that (4.5) is true when $p=0$.

If $p \geqq 1$, it is only necessary to consider the formal product of (1.1) and $g(x)$ with either $\lambda=1, \mu=0$ or $\lambda=0, \mu=1$. In the first case the constant term of the new series is $a_{p} / 2$ and, in the second, it is $b_{p} / 2$. Hence (4.5) and (4.6) are true for $p \geqq 1$.

5. Upper and lower sums $(C, k)$. Let $S^{k}(x)$ and $s^{k}(x)$ denote the $\lim$ sup and $\lim$ inf, respectively, as $n \rightarrow \infty$, of $A_{n}^{k}(x) / E_{n}^{k}$. In order to extend the results of $\S 4$ to the case where (1.1) is not summable for every $x$, some information is needed concerning the behavior of $S^{k}(x)$ and $s^{k}(x)$ at points of non-summability.

LeMma 5.1. Let $P$ denote the set of points $x$ for which

$$
-\infty<s^{k}(x) \leqq S^{k}(x)<+\infty \text {. }
$$


Then $P$ is a set $F_{\sigma}$.

Proof. Let $S_{n}^{k}(x)$ and $s_{n}^{k}(x)$ denote the sup and the inf, respectively, of the set $A_{m}^{k}(x) / E_{m}^{k}$ for $m \geqq n$. Then $S_{n}^{k}(x)$ is a monotone decreasing sequence of lower semicontinuous functions with limit $S^{k}(x)$ and $s_{n}^{k}(x)$ is a monotone increasing sequence of upper semicontinuous functions with limit $s^{k}(x)$. If $P_{n}$ denotes the set of points $x$ for which $S_{n}^{k}(x)-s_{n}^{k}(x) \leqq n$, then each $P_{n}$ is closed and, clearly,

$$
P=\bigcup_{n=1}^{\infty} P_{n} .
$$

LEMMA 5.2. If condition (3.3) is satisfied and $F(x)$ is the function of Theorem 3.1, let $4^{k+2} F(x)$ and $\delta^{k+2} F(x)$ denote the lim sup and lim inf, respectively, as $h \rightarrow 0$ of (3.1) if $k+2=2 m$ and of (3.2) if $k+2=2 m$ +1. Then $\Delta^{k+2} F(x)$ and $\delta^{k+2} F(x)$ lie in the interval

$$
\left(\varphi^{k}(x)-C_{k} \psi^{k}(x), \varphi^{k}(x)+C_{k} \psi^{k}(x)\right),
$$

where

$$
2 \varphi^{k}(x)=S^{k}(x)+s^{k}(x), \quad 2 \psi^{k}(x)=S^{k}(x)-s^{k}(x),
$$

and $C_{k}$ depends only on $k$.

Proof. This result is equivalent (when $k+2=2 m$ ) to [11, Theorem B] and it may also be proved by the method of $[13, \S 10.42]$.

THEOREM 5.1 Suppose that the series (1.1) is summable $(C, k)$ to a finite function $f(x)$ for all $x \in[0,2 \pi]-E$, where $E$ is at most countable. Then the set $S$ of points $x$ for which either $\Delta^{k+2} F(x)=+\infty$ or $\delta^{k+2} F(x)$ $=-\infty$ is a scattered set (clairsemé, [3, p. 90]), that is, a set which contains no subset that is dense-in-itself.

Proof. It follows from (3.7) with $r=0$ that $S \subset E$ so that $S$ is at most countable. By Lemma 5.2, $S$ is the complement of the set $P$ and is therefore a set $G_{\delta}$. But a set $G_{\delta}$ that is at most countable cannot contain a subset that is dense-in-itself [5, VIII, p. 136].

6. A new definition of the $P^{k+2}$-integral. Since only the case $k=$ $2 m-2$ is considered in detail in [7], the same restriction will be made in this section. Two of the requirements for a major function $Q(x)$ and a minor function $q(x)$ are [7, Definition 5.1]

$$
\begin{array}{ll}
\delta^{2 m} Q(x) \geqq f(x) \geqq \Delta^{2 m} q(x) & x \in(a, b), \\
\delta^{2 m} Q(x) \neq-\infty, \quad \Delta^{2 m} q(x) \neq+\infty & x \in(a, b) .
\end{array}
$$

Under the hypotheses of Theorem 5.1, the function $Q(x)$ defined by (4.1) satisfies (6.1) only for $x \in[0,2 \pi]-E$, where $E$ is at most countable, 
and (6.2), only for $x \in[0,2 \pi]-S$, where $S$ is scattered. In order to extend the results of $\S 4$, it must be possible to weaken (6.1) and (6.2) and still define an integral.

It is not difficult to see that there is no change in the $P^{2 m}$-integral if $(6.1)$ holds only for $x \in(a, b)-E_{0}$, where $E_{0}$ is of measure zero. The method is similar to that used for the Perron integral and the $P^{2}$-integral [6, Theorem 3.1]. The modification of (6.2) is not quite so easy.

If the reasons for requiring (6.1) and (6.2) are examined, it is seen that they are needed to make sure that the difference

$$
\delta^{2 m} Q(x)-\Delta^{2 m} q(x)
$$

is defined and nonnegative for $x \in(a, b)$. This, in turn, goes back to [7, Theorem 4.1] which gives sufficient conditions that a continuous function $F(x)$ should satisfy in order that $D^{2 m-2} F(x)$ should be convex. For convenience, the theorem is restated here :

If $F(x)$ satisfies conditions $A_{2 m}$ and $B_{2 m-2}[7, \S 2]$ in $(a, b)$ and if

$$
\Delta^{2 m} F(x)>0 \quad x \in(a, b),
$$

then $D^{2 m-2} F(x)$ is convex and, for $1 \leqq r \leqq m-1$, each $D^{2 m-2 r} F(x)$ is cont $i$ nuous in $(a, b)$.

It is therefore necessary to establish the conclusions of this theorem under weaker hypotheses.

Lemma 6.1. The conclusions of [7, Theorem 4.1] remain true if (6.3) is replaced by $\Delta^{2 m} F(x) \geqq 0, x \in(a, b)$.

Proof. For each positive integer $n$, let

$$
F_{n}(x)=F(x)+x^{2 m} / n(2 m) ! .
$$

Then, for $1 \leqq r \leqq m-1$,

$$
D^{2 m-2 r} F_{n}(x)=D^{2 m-2 r} F(x)+x^{2 m-2 r} / n(2 m-2 r) !
$$

and

$$
\Delta^{2 m} F_{n}(x)=\Delta^{2 m} F(x)+1 / n>0 .
$$

Thus $F_{n}(x)$ satisfies all the original conditions of [7, Theorem 4.1]. Since each $D^{2 m-2 r} F_{n}(x)$ tends uniformly to $D^{2 m-2 r} F(x)$ the same conclusions hold for $D^{2 m-2 r} F(x)$.

THeOREM 6.1. If in the hypotheses of [7, Theorem 4.1], the condition (6.3) is replaced by the two conditions

$$
\Delta^{2 m} F(x) \geqq 0 \quad x \in(a, b)-S,
$$


where $S$ is scattered, and

$$
\underset{h \rightarrow 0}{\limsup } h \theta_{2 m}(x, h) \geqq 0 \geqq \liminf _{h \rightarrow 0} h \theta_{2 m}(x, h) \quad x \in S,
$$

then the conclusions remain true.

Proof. Let $H$ denote the set of points $x$ in $(a, b)$ for which $A^{2 m} F(x)$ $<0$. From (6.4) it follows that $H \subset S$, and if $H$ is not empty, it must contain at least one isolated point $x_{0}$ and there is an interval $(\alpha, \beta)$ containing $x_{0}$ but no other points of $H$. All the conditions of Lemma 6.1 are satisfied in each of the intervals $\left(\alpha, x_{0}\right),\left(x_{0}, \beta\right)$ and the conclusions are valid in each interval. By [7, Lemma 3.4] they are also valid in each of the closed intervals $\left[\alpha, x_{0}\right],\left[x_{0}, \beta\right]$.

Therefore, for the function $\rho(x)=D^{2 m-2} F(x)$, the difference quotient $\left\{\rho\left(x_{0}\right)-\rho\left(x_{0}-h\right)\right\} / h$ is monotone increasing as $h \rightarrow 0$. Hence the left-hand derivative $\rho_{-}^{\prime}(x)$ exists (possibly equal to $+\infty$ ), and, similarly, so does the right-hand derivative $\rho_{+}^{\prime}\left(x_{0}\right)$ (possibly equal to $-\infty$ ). Then, since

$$
\begin{gathered}
2 h \theta_{2}\left(\rho ; x_{0}, h\right)=\left\{\rho\left(x_{0}+h\right)+\rho\left(x_{0}-h\right)-2 \rho\left(x_{0}\right)\right\} / h, \\
\lim _{h \rightarrow 0} 2 h \theta_{2}\left(\rho ; x_{0}, h\right)=\rho_{+}^{\prime}\left(x_{0}\right)-\rho_{-}^{\prime}\left(x_{0}\right) .
\end{gathered}
$$

But, since each $D^{2 m-2 r} F(x)$ is continuous in $[\alpha, \beta]$, it follows [11, Lemma 7] that $F(x)$ has continuous ordinary derivatives $F^{(s)}(x), 1 \leqq s$ $\leqq 2 m-2$, and in particular, $F^{(2 m-2)}(x)=D^{2 m-2} F(x)$. Hence, from (6.5) by repeated application of the theorem on indeterminate forms,

$$
\limsup _{h \rightarrow 0} h \theta_{2}\left(\rho ; x_{0}, h\right) \geqq 0 \geqq \liminf _{h \rightarrow 0} h \theta_{2}\left(\rho ; x_{0}, h\right) .
$$

Then, from (6.6), $\rho_{+}^{\prime}\left(x_{0}\right)=\rho_{-}^{\prime}\left(x_{0}\right)$ and $\rho(x)$ is smooth at $x=x_{0}$. It follows $[13, \S 11.31]$ that $\rho(x)$ is convex in $[\alpha, \beta]$ and, in particular, that $\delta^{2} \rho\left(x_{0}\right)$ $\geq 0$. But then, by another application of the theorem on indeterminate forms,

$$
\Delta^{2 m} F\left(x_{0}\right) \geqq \delta^{2 m} F\left(x_{0}\right) \geqq \delta^{2} \rho\left(x_{0}\right) \geqq 0,
$$

which contradicts the fact that $x_{0} \in H$. Hence $H$ must be empty and $\Delta^{2 m} F(x) \geqq 0$ in $(a, b)$. The conclusions of Theorem 6.1 now follow from Lemma 6.1.

Once Theorem 6.1 (replacing [7, Theorem 4.1]) has been established, there is no further difficulty in defining a modified $P^{2 m}$-integral. The only difference between the new and old definitions is that (6.4) and (6.5) are replaced by

$$
\delta^{2 m} Q(x) \geqq f(x) \geqq \Delta^{2 m} q(x) \quad x \in(a, b)-E_{0},
$$

where $E_{0}$ is of measure zero, and 


$$
\delta^{2 m} Q(x) \neq-\infty, \quad \Delta^{2 m} q(x) \neq+\infty \quad x \in(x, b)-S,
$$

where $S$ is scattered, provided that $Q(x)$ and $q(x)$ satisfy (6.5).

The final result is the following:

THEOREM 6.2. Suppose that the series (1.1) is summable $(C, k)$ to a finite function $f(x)$ for all $x \in[0,2 \pi]-E$, where $E$ is at most countable, and let $f(x)=0, x \in E$. If $A_{n}^{k-1}(x)=o\left(n^{k}\right)$ for $x \in E$ and $B_{n}^{k-1}(x)=o\left(n^{k}\right)$ for $x \in[0,2 \pi]$, then $f(x), f(x) \cos p x, f(x)$ sin $p x$ are each $P^{k+2}$-integrable and the coefficients of (1.1) are given by (4.5) and (4.6).

7. Remark on the $P^{2}$-integral. It was noted in $[7, \S 6]$ that the $P^{2 m}$-integral for $m=1$ was possibly not the same as the original $P^{2}$-integral. It is, however, not difficult to see that the new $P^{2 m}$-integral for $m=1$ is the same as the original. The reason is that the set of points where $\delta^{2} Q(x)=-\infty$ or $\Delta^{2} q(x)=+\infty$ is a set $G_{\delta}$ and, if at most countable, it must be scattered.

\section{REFERENCES}

1. J. C. Burkill, The expression of trigonometrical series in Fourier form, J. London Math. Soc., 11 (1936), 43-48.

2. - Integrals and trigonometric series, Proc. London Math. Soc. (3) 1 (1951), $46-57$.

3. A. Denjoy, Leçons sur le calcul des coefficients d'une série trigonométrique, Paris, 1941 and 1949.

4. G. H. Hardy, Divergent series, Oxford, 1949.

5. F. Hausdorff, Mengenlehre, Berlin, 1927.

6. R. D. James, A generalized integral II, Canadian J. Math., 2 (1950) 297-306.

7. __. Generalized n-th primitives, Trans. Amer. Math. Soc., 76 (1954), 149-176.

8. J. Marcinkiewicz and A. Zygmund, On the differentiability of functions and summability of trigonometric series, Fund. Math., 26 (1936), 1-43.

9. L. M. Milne-Thomson, The calculus of finite differences, London, 1933.

10. S. Verblunsky, On the theory of trigonometric series VII, Fund. Math., 23 (1934), 193-235.

11. F. Wolf, Summable trigonometric series: an extension of uniqueness theorems, Proc. London Math. Soc. (2) 45 (1939), 328-356.

12. A. Zygmund, Sur la théorie riemannienne des séries trigonométriques, Math. Z., 24 (1926), 47-104.

13. _ Trigonometrical series, Warsaw, 1935.

University of British Columbia and Michigan State College 


\section{PACIFIC JOURNAL OF MATHEMATICS}

\section{EDITORS}

H. L. Royden

Stanford University

Stanford, California

E. HewitT

University of Washington

Seattle 5 , Washington
R. P. Dilworth

California Institute of Technology Pasadena 4, California

E. G. Straus

University of California

Los Angeles 24, California

\section{ASSOCIATE EDITORS}

E. F. BECKENBACH

C. E. BURGESS

H. BUSEMANN

H. FEDERER

\author{
M. HALL \\ P. R. HALMOS \\ V. GANAPATHY IYER \\ R. D. JAMES
}

M. S. KNEBELMAN

I. NIVEN

T. G. OSTROM

M. M. SCHIFFER
J. J. STOKER

G. SZEKERES

F. WOLF

K. YOSIDA

\section{SUPPORTING INSTITUTIONS}

UNIVERSITY OF BRITISH COLUMBIA

CALIFORNIA INSTITUTE OF TECHNOLOGY

UNIVERSITY OF CALIFORNIA

MONTANA STATE UNIVERSITY

UNIVERSITY OF NEVADA

OREGON STATE COLLEGE

UNIVERSITY OF OREGON

UNIVERSITY OF SOUTHERN CALIFORNIA
STANFORD UNIVERSITY

UNIVERSITY OF UTAH

WASHINGTON STATE COLLEGE

UNIVERSITY OF WASHINGTON

AMERICAN MATHEMATICAL SOCIETY CALIFORNIA RESEARCH CORPORATION HUGHES AIRCRAFT COMPANY 


\section{Pacific Journal of Mathematics}

\section{Vol. 6, No. $1 \quad$ November, 1956}

David Blackwell, An analog of the minimax theorem for vector payoffs..... 1

L. W. Cohen, A non-archimedian measure in the space of real

sequences ..................................... 9

George Bernard Dantzig, Constructive proof of the Min-Max theorem ..... 25

Jim Douglas, On the numerical integration of quasilinear parabolic

differential equations ............................... 35

James Michael Gardner Fell, A note on abstract measure ............. 43

Isidore Isaac Hirschman, Jr., A note on orthogonal systems . . . . . . . . . . 47

Frank Harary, On the number of dissimilar line-subgraphs of a given

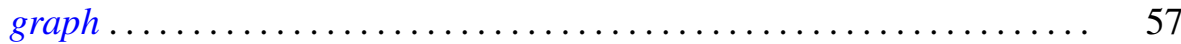

Newton Seymour Hawley, Complex bundles with Abelian group......... 65

Alan Jerome Hoffman, Morris Newman, Ernst Gabor Straus and Olga

Taussky, On the number of absolute points of a correlation ...........

Ernst Gabor Straus and Olga Taussky, Remark on the preceding paper.

Algebraic equations satisfied by roots of natural numbers . . ........ 97

Ralph D. James, Summable trigonometric series ................. 99

Gerald R. Mac Lane, Limits of rational functions . . . . . . . . . . . . . . . 111

F. Oberhettinger, Note on the Lerch zeta function ................. 117

Gerald C. Preston, On locally compact totally disconnected Abelian groups and their character groups ........................... 121

Vikramaditya Singh and W. J. Thron, On the number of singular points, located on the unit circle, of certain functions represented by

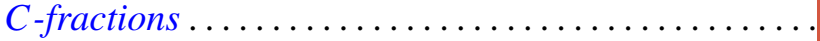

Sherman K. Stein, The symmetry function in a convex body ... 145 Edwin Weiss, Boundedness in topological rings.............

Albert Leon Whiteman, A sum connected with the series for the partition

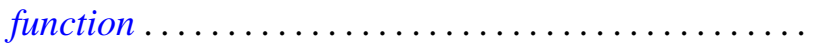

Alfred B. Willcox, Some structure theorems for a class of Banach algebras.

Joseph Lawrence Zemmer, Some remarks on p-rings and their Boolean geometry... 